\title{
MODELO DE PROGRAMAÇÃO MATEMÁTICA PARA A OTIMIZAÇÃO DO PLANEJAMENTO DE PRODUÇÃO E SUSBSTITUIÇÃO DE INJETORAS NA INDÚSTRIA DE ELETRODOMÉSTICOS
}

\author{
Anibal Alberto Vilcapoma Ignacio \\ Universidade Federal Fluminense - UFF \\ anibalvilcapoma@gmail.com \\ Léa Maria Dantas Sampaio \\ COPPE/UFRJ \\ Centro de Tecnologia - Cidade Universitária - \\ I. do Fundão - Rio de Janeiro - RJ \\ leasampaio@gmail.com \\ Ruan Carlos Alves Pereira \\ Universidade Federal Fluminense - UFF \\ ruancap@id.uff.br \\ Pedro Alan Souza Rodrigues Silva \\ Universidade Federal Fluminense - UFF \\ uff.pedro@gmail.comr
}

\section{Resumo}

O presente trabalho faz uma análise das variáveis de uma linha produtiva de materiais plásticos para eletrodomésticos, a partir da utilização de injetoras, indicando o melhor momento e de que forma deve ser feita a troca destes equipamentos. Neste estudo utilizam-se previsões de demandas já conhecidas pela empresa, que vem a ser um dos itens que ajuda a compor a programação da produção. É dado um modelo de programação matemática para solucionar problemas de planejamento deste tipo de produção.

Palavras-chave: Programação matemática, Planejamento da produção, Indústria de eletrodomésticos.

\begin{abstract}
This paper analyzes the variables of a productive line of plastics for appliances, from the use of injection, indicating the best time and how the exchange of such equipment must be made. In this study data are used to forecast demands already known by the company, which happens to be one of the items that helps make the production schedule. A model of mathematical programming is given to solve planning problems of this type of production.
\end{abstract}

Keywords: Mathematical Programming, Production Planning, appliance industry 


\section{INTRODUÇAO}

Grande número de fatores, considerados no planejamento operacional das empresas, cria uma complexidade relacionada à aleatoriedade natural das demandas e as múltiplas decisões de natureza combinatória que devem ser tomadas nos diversos processos decisórios e que geram incertezas sobre os resultados esperados no planejamento. As atividades de planejamento de médio prazo são especialmente importantes em empresas de manufatura, uma vez que permite dimensionar recursos produtivos que terá impacto no atendimento da demanda.

Conforme aumenta o número, tamanho e a complexidade das estruturas produtivas, mais importante se tornam o impacto do gerenciamento sobre a rentabilidade dos negócios. $\mathrm{O}$ planejamento da produção e a identificação de momentos de renovação da estrutura produtiva é uma decisão importante e está relacionada com o custo operacional de cada uma delas. A questão é qual o momento ideal de substituição dos equipamentos, levando em conta aspectos como ciclo de vida do equipamento e sua obsolescência em relação às novas tecnologias presentes no mercado.

Por outro lado, a gestão da manutenção neste contexto, se torna relevante já que o objetivo desta é determinar, antecipadamente, possível necessidade de correção de problemas, minimizando o tempo de parada a fim de aumentar a disponibilidade do equipamento e maximizar o seu ciclo de vida. Embora a manutenção traga benefícios ao seu sistema produtivo, seu custo pode ser elevado, já que exige constante monitoramento dos equipamentos específicos e o emprego de profissionais especializados. Com isso, ao se verificar que a vida útil de um equipamento foi atingida, sua substituição se faz necessária. Contudo, de acordo com Souza e Clemente [1], geralmente, as empresas orientam-se por aspectos técnicos para a tomada de decisão, a respeito de possíveis baixas e/ou substituições de equipamentos, o que no longo prazo é insustentável, pois os gastos com manutenção se tornam altos e deixam de ser efetivos.

A indústria de eletrodomésticos/utilidades no Brasil cresceu 23\% em 2012, segundo a associação brasileira da indústria elétrica e eletrônica. $\mathrm{O}$ processo produtivo dos eletrodomésticos/utilidades está formado por dois tipos de componentes: os componentes pré fabricadas por outros segmentas da industria, como por exemplo motores, parafusos, transformadores, etc. e os fabricados pela própria industria de eletrodomésticos, como as carcaças de polipropileno. Estes dois tipos de peças logo são montadas para obter o produto final.

O processo de injeção está fortemente ligado ao processo produtivo da empresa, mesmo que em algumas delas ele também faça parte de componentes que podem ser terceirizadas. Pelo fato do processo de injeção evoluir continuamente, aparecem no mercado novos tipos de injetoras com maior capacidade de produção, menor custo operacional e maior eficiência. Contudo, elas custam bem mais caro e sua substituição deve ser formulada, a partir de análises de sua viabilidade técnico-econômica. O desgaste também é um fator importante a ser avaliado, segundo Hirschfeld [2], pois é típico dos equipamentos, cuja eficiência decresce gradativamente, com o tempo ou com o uso, provocando aumento nos custos operacionais e de manutenção, além de prejudicar a qualidade do serviço realizado, diminuindo a sua eficiência produtiva. Estas questões são tratadas do um ponto de vista econômico-financeiro, porem, no âmbito do planejamento de produção, a substituição dos equipamentos é feita gradualmente e não de forma total. A substituição gradual dos equipamentos é imposta muitas vezes por limitações financeiras da organização ou pela existência de demanda futura a ser atendida no mercado.

O presente trabalho faz uma análise das variáveis de uma linha produtiva de materiais plásticos para eletrodomésticos, a partir da utilização de injetoras, indicando o planejamento ótimo da produção, o melhor momento desta e a forma que deve ser feita a troca 
destes equipamentos. Neste estudo utilizam-se previsões de demandas já conhecidas pela empresa, o que vem a ser um dos itens de ajuda para se compor a programação da produção.

Diversos trabalhos a respeito desse tema podem ser encontrados na literatura, tais como Fleischmann [3], que utiliza modelos de otimização para solucionar tais questões.

Segundo Manrich [4], é muito importante conhecer quais são os pontos nevrálgicos de um processo e como certos parâmetros podem ser alterados para modificar a qualidade de um produto final, o que pode ser, muitas vezes, adquirido através de alguma ferramenta de pesquisa operacional ou software computacional.

Um dos poucos métodos exatos para se resolver o problema de programação da produção, com tempo de troca dependente da sequência, é encontrado em Haase \& Kimms [5]. Pela dificuldade computacional métodos heurísticos foram desenvolvidos, como descritas em Meyr ([6] e [7]).

Sistemas produtivos em paralelo com demanda constante são encontrados em Carreno [8]. O trabalho de De Matta \& Guignard [9] trata o problema de programação da produção com máquinas paralelas com horizonte rolante.

Em casos em que não seja possível programar a produção de alguns itens de forma a atender completamente a suas demandas num certo período, a empresa renegocia os prazos de entrega com os clientes de forma a minimizar seu descumprimento. Portanto, uma melhor programação da produção reduz o número de renegociações de prazos de entrega entre a empresa e seus clientes. Além disso, também facilita a aplicação de certas técnicas de programação, como o uso de "horizonte rolante" (CLARK \& CLARK [10]).

\section{MÁQUINAS INJETORAS DE TERMOPLÁSTICOS}

A moldagem por injeção, ilustrado na Figura 1, foi inicialmente desenvolvida para a transformação de resinas termoendurecíveis, como ureia formaldeído (comercialmente conhecido como Baquelita). O processo teve um enorme desenvolvimento com o advento dos materiais termoplásticos que se verificou após a Segunda Guerra Mundial.

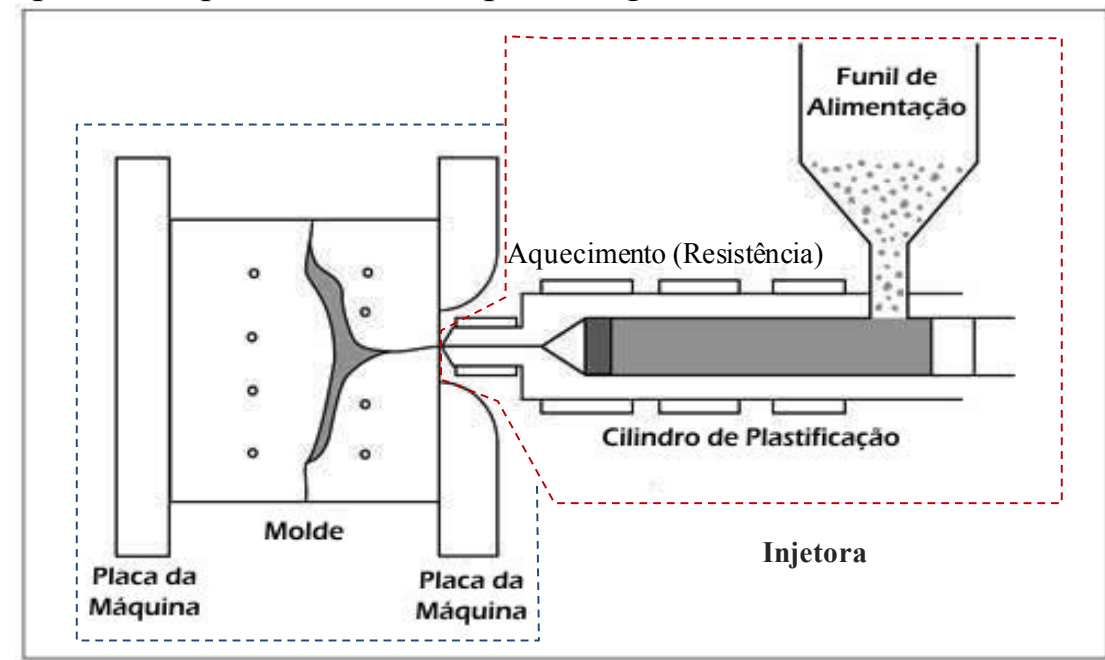

Figura 1 - Moldagem por injeção (Fonte: Dupont Telesolutions)

Atualmente, a moldagem por injeção é um dos principais processos de transformação de materiais de base polimérica, tendo enorme importância nos grandes mercados consumidores como: embalagens, construção civil, automóvel e material elétrico e eletrônico.

O grande sucesso desta tecnologia deve-se ao efeito combinado de várias vantagens comparativas, entre as quais se salientam: elevada produção, grande reprodutibilidade e precisão dimensional e grande flexibilidade em termos de geometria e dimensões das moldagens. Podem ser moldados por injeção os seguintes produtos: termoplásticos, termo fixos e elastômeros vulcanizados. A moldagem por injeção também pode ser utilizada para a fabricação de peças cerâmicas ou metálicas, a partir de compostos que utilizam um material polimérico como vetor. 
A moldagem por injeção de termoplásticos obedece às etapas típicas de transformação destes materiais, envolvendo sequencialmente as etapas seguintes: (i) aquecimento do material, até este adquirir uma viscosidade suficientemente baixa; (ii) conformação sobre pressão; e (iii) resfriamento com consequente recuperação da rigidez. O molde é uma estrutura normalmente metálica, na qual a injetora introduz através de um sistema hidráulico as resinas termoplásticas aquecidas à alta temperatura (as temperaturas variam entre $215^{\circ} \mathrm{C}$ e $300^{\circ} \mathrm{C}$ ). As pressões de injeção variam entre 15 a $140 \mathrm{Mpa}$. Depois de injetadas no molde, é necessário um tempo de esfriamento para dar consistência às resinas aquecidas. Após isso se pode abrir o molde, se retirar as peças e novamente se repetir o ciclo de injeção. Um mesmo molde pode ser utilizado em um conjunto de injetoras e este pode ser realocado a uma determinada injetora, quando necessário. Vale ressaltar que existem diversos parâmetros de eficiência nas injetoras como, por exemplo, o potencial de injeção (o que restringe uma gama de moldes que podem ser usados e, por consequência, restringe os produtos a serem fabricados).

Cada injetora apresenta determinado custo operacional, em termos de consumo de energia. Normalmente, injetoras de ciclo de produção menor utilizam um consumo maior de energia. A troca de moldes entre duas injetoras tem custos de instalação diferentes, posto que por suas características técnicas fazem-se necessários limpeza e ajuste de parâmetros de produção. Estes diversos aspectos dificultam o planejamento de produção e as políticas de substituição e modernização das injetoras.

\section{DESCRIÇÃO DO PROBLEMA}

Normalmente a indústria de eletrodomésticos no Brasil é formada por uma estrutura de injeção de polipropileno e um sistema de montagens de peças já fabricadas por outras indústrias. Como exemplo tem-se que, para fabricar um ventilador, as carcaças e as hélices do mesmo são fabricadas pela injetora, enquanto o motor, os parafusos e os demais componentes são apenas montados. O processo da montagem inclui a pintura e a embalagem do produto.

Portanto, a estrutura de produção é formada basicamente por injetoras e moldes com os quais são construídas as peças. Neste sistema produtivo existem basicamente dois tipos de estoques: os estoques de peças para fabricação de produtos e os de produtos acabados.

A demanda por produtos eletrodomésticos segue um comportamento caracterizado pela natureza do produto. Por exemplo, a demanda por ventilador, do mês de setembro até dezembro é motivada pelo verão, enquanto que outros produtos não apresentam esse comportamento, como é o caso do liquidificador, cujo gráfico da demanda está ilustrado na Figura 2.

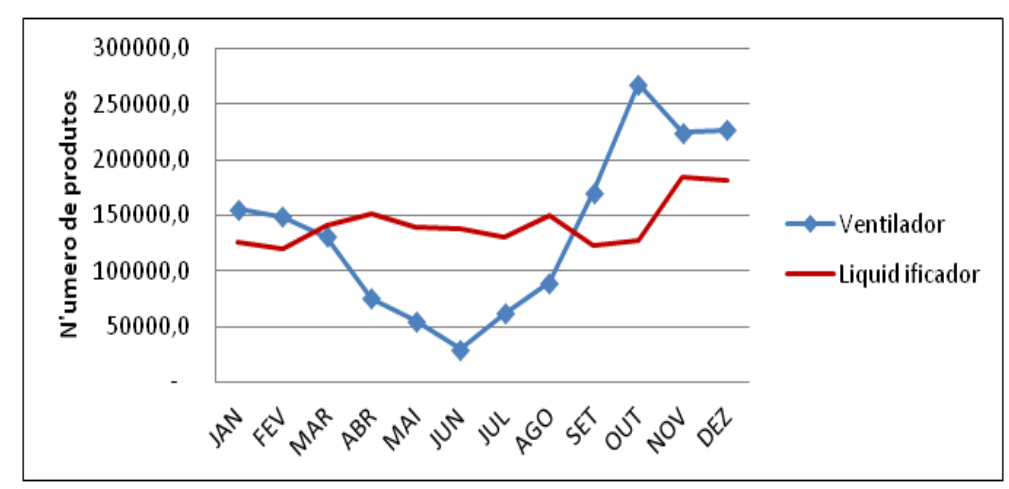

Figura 2 - Ciclo de demanda de produtos

Dada esta situação, existe um problema de planejamento da produção anual, de forma a suprir a demanda. É importante lembrar que este tipo de empresa fabrica diversos tipos de produtos em número muito grande, durante todo o ano, de forma a fazer frente às oscilações da demanda e com o uso, tanto dos estoques de produtos acabados quanto de peças para fabricação. 
O segundo problema que enfrenta este tipo de indústria é quando o ciclo de vida das injetoras está no fim e a obsolescência destes equipamentos ocasionam um custo operacional e um alto nível de ineficiência, tais como: uso excessivo de eletricidade, elevado tempo de injeção, desperdício e elevado tempo de setup de máquina.

Dado esta situação existe um problema de planejamento da produção por ano de forma a suprir a demanda. O problema então pode ser resumido da seguinte forma: Dado que existe um conjunto de:

1. Demandas mensais de diversos produtos durante o ano;

2. Injetoras com diversas capacidades e custos operacionais;

3. Moldes que podem fabricar as peças de cada um dos produtos;

4. Recursos de montagem das peças para a produção.

Busca-se definir:

- Um plano de produção anual para cada um dos produtos e

- A alocação de moldes e injetoras que minimize o custo operacional deste planejamento.

Cabe ressaltar que o conjunto de injetoras é formado por aquelas que fazem parte da capacidade produtiva. No entanto, a empresa as considera possíveis injetoras a serem substituídas. Este problema é mostrado na Figura 3.

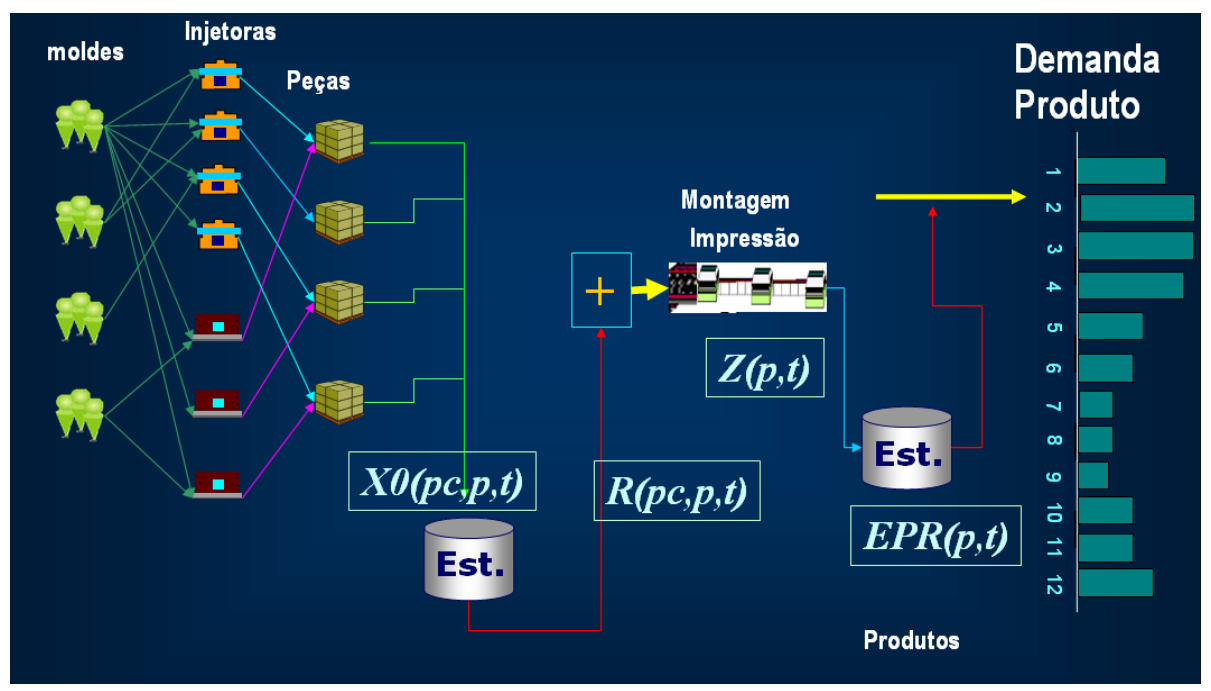

Figura 3 - Estrutura do processo produtivo de injetoras

Uma característica que se destaca no processo produtivo deste tipo de indústria é que a produção da maioria dos itens se orienta por flutuações da demanda (ver Figuras 4 e 5).

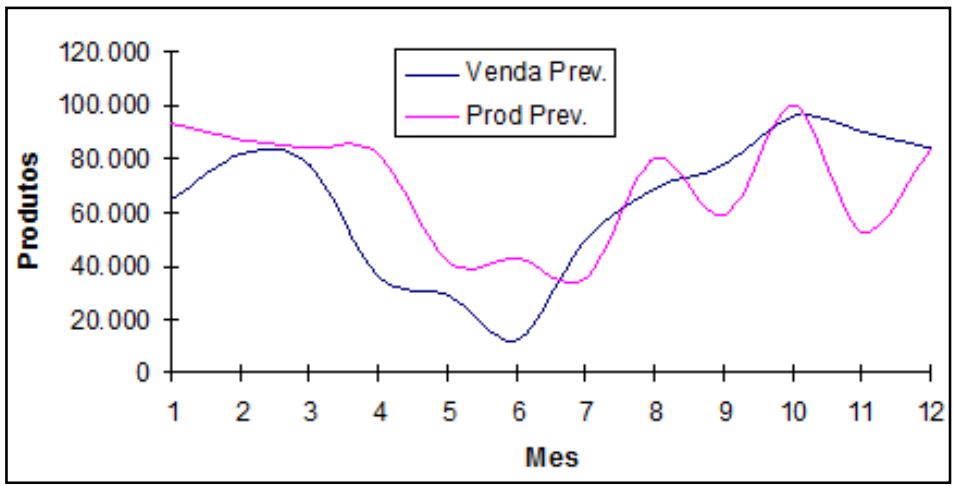

Figura 4. Demanda e produção previstas de ventiladores 
$\mathrm{Na}$ Figura 4 mostra a flutuação da demanda, referente à produção prevista de ventiladores e na Figura 5, de liquidificadores.

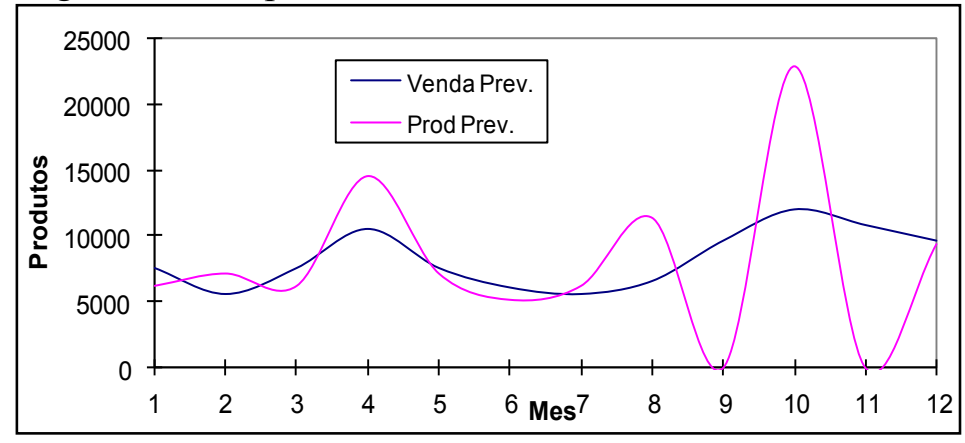

Figura 5 - Demanda e produção previstas de liquidificadores

Este tipo de política naturalmente é realizado pela complexidade do processo produtivo, uma vez que se deve lidar com diversos tipos de produtos, vários tipos de injetoras e moldes com características diversas de capacidades e custos associados. Tentar seguir as flutuações de demanda leva a se ter um custo de instalação muito alto, considerando-se tempo e custos, alem de não se fazer uso eficiente das políticas de estoques, tanto de peças quanto de produtos.

Índices

A seguir é apresentada a formulação do modelo de otimização para o problema.

$p$ : conjunto de produtos;

$t$ : intervalos de tempo;

$i$ : conjunto de moldes $(p c=i)$;

$j$ : conjunto de injetoras (Num_j: número de injetoras);

Parâmetros

$p c$ : conjunto de peças;

$\boldsymbol{D}(\boldsymbol{p}, \boldsymbol{t})$ : Demanda do produto $\mathrm{p}$ no tempo t.

$\boldsymbol{C E S P R}(\boldsymbol{p})$ : Custo unitário de estoque do produto $\mathrm{p}$ em um intervalo $\mathrm{t}$.

$\boldsymbol{C M T}(\boldsymbol{p})$ : Custo unitário da montagem do produto p. Este custo somente considera a mão de obra do processo da montagem.

$\boldsymbol{C I M}(\boldsymbol{p})$ : Custo unitário da impressão do produto $\mathrm{p}$.

$\boldsymbol{C E S P C}(\boldsymbol{p} \boldsymbol{c})$ : Custo unitário de estoque da peça pc.

$\boldsymbol{C S e t u p}(\boldsymbol{p c})$ : Custo de setup da peça pc.

$\boldsymbol{C E N E R}(\boldsymbol{p} \boldsymbol{c}, \boldsymbol{j})$ : Custo de energia de cada peca injetada $p c$ por a injetora $\mathrm{j}$.

$\boldsymbol{C M A I N}(\boldsymbol{j})$ : Custo unitário da mão de obra por injetora.

$\boldsymbol{C M A N U}(\boldsymbol{j})$ : Custo fixo de manutenção por injetora.

$\boldsymbol{C C A P}$ : Custo de capital para o calculo do estoque de produtos acabados ( $2 \%$ ao mês).

$\boldsymbol{N u m \_} \boldsymbol{p c}(\boldsymbol{p c}, \boldsymbol{p})$ : Número de pecas do conjunto pc utilizado no produto $\mathrm{p}$.

$\boldsymbol{P V}(\boldsymbol{p}, \boldsymbol{t})$ : Preço de venda do produto $\mathrm{p}$ no tempo t.

$\boldsymbol{C M A P}(\boldsymbol{p}, \boldsymbol{t})$ : Preço da matéria-prima do produto $\mathrm{p}$ no tempo t.

$\boldsymbol{C a p} \boldsymbol{M} \boldsymbol{M} \boldsymbol{n}_{-} \boldsymbol{E} \boldsymbol{P} \boldsymbol{R}(\boldsymbol{p})$ : Capacidade mínima de produto $\mathrm{p}$ em estoque.

$\boldsymbol{C a p} \boldsymbol{M a x} \_\boldsymbol{E P R}(\boldsymbol{p})$ : Capacidade máxima do produto $\mathrm{p}$ em estoque.

Cap_Min_EPC $(p c$ :Capacidade mínima da peça $p c$ em estoque. Preliminarmente é estipulado como o montante referente a dois dias garantidos para montagem.

$\boldsymbol{C a p} \boldsymbol{M a x} \_\boldsymbol{E} \boldsymbol{C}(\boldsymbol{p c})$ : Capacidade máxima da peça $p c$ em estoque.

$\boldsymbol{C a p} \boldsymbol{I} \boldsymbol{N} \boldsymbol{J}(\boldsymbol{p} \boldsymbol{c}, \boldsymbol{j})$ : Capacidade da injeção de uma peça $p c$ na injetora j. Este valor é considerado como sendo o $85 \%$ de 24 horas x 26 dias úteis x Tarefa de cada peça. 
Cap_MT(p): Capacidade de montagem do produto p. Devem ser consideradas 7.83 horas por dias multiplicadas por cada tarefa de cada produto.

Cap_IM(p): Capacidade de impressão do produto p. Inicialmente é considerada uma capacidade ilimitada.

\section{Variáveis}

$\boldsymbol{S}(\boldsymbol{d}, \boldsymbol{p})$ : Quantidade de produtos acabados que saem do estoque para o atendimento da demanda.

$\boldsymbol{Z}(\boldsymbol{p}, \boldsymbol{d})$ : Quantidade produzida de produto p que é estocada.

$\boldsymbol{E P R}(\boldsymbol{p}, \boldsymbol{t}):$ Quantidade de produtos $\mathrm{p}$ em estoque, no final do período $\mathrm{t}$.

$\boldsymbol{E P R}(\boldsymbol{p c}, \boldsymbol{t})$ : Quantidade de peças pc em estoque, no final do período $t$.

$$
\begin{aligned}
& Y(j)=\left\{\begin{array}{l}
1 \text { Se a injetora } j \text { é utilizada } \\
0
\end{array} \quad\right. \text { c.c } \\
& X(p c, j)=\left\{\begin{array}{lr}
1 & \text { Se o molde pc é alocado a injetora } j \\
0 & \text { c.c }
\end{array}\right.
\end{aligned}
$$

$\mathrm{X} 1(\mathrm{pc}, \mathrm{p}, \mathrm{t})$ e X0(pc,p,t): Quantidade de peças injetadas, que são usadas para fabricação e estoque, respectivamente.

\section{Modelo}

$$
\begin{aligned}
\text { Maximizar: } & \sum_{p} \sum_{t}(S(p, t))^{*} P V(p) \\
& -\sum_{p} \sum_{t}(\operatorname{CMAP}(p)+\operatorname{CMT}(p)+\operatorname{CIM}(p))^{*} Z(p, t) \\
& -\sum_{p c} \sum_{j} \operatorname{CSetup}(p c, j)^{*} X(i, j) \\
& -\sum_{p c} \sum_{p, t} \operatorname{CMAIN}(p c)^{*}(X 1(p c, p, t)+X 0(p c, p, t)) \\
& -\sum_{j} \sum_{p c} \sum_{p, t}(C E N E R(p c, j))^{*}(X 1(p c, p, t)+X 0(p c, p, t)) \\
& -\sum_{p, t} \operatorname{EPR}(p, t)^{*} P V(p, t)^{*} C C A P-\sum_{p c, p, t} \operatorname{EPC}(p c, p, t)^{*} C E S P C(p c) \\
& -\sum_{j} \operatorname{CMANU}(j)^{*} Y(j)
\end{aligned}
$$

A primeira componente da função objetivo representa o faturamento total com os produtos acabados, a segunda componente da função objetivo representa o custo com a matéria prima, a montagem e a impressão, no nível de produto acabado. A terceira componente representa o custo de setup de um molde em uma determinada injetora. A quinta e sexta componentes representam, respectivamente, o custo de mão de obra e custo de energia por cada peça fabricada. A sétima e oitava componentes apresentam, respectivamente, os custos de estoque de produtos e de peças. A última expressão representa o custo de manutenção.

\section{Restrições:}

$S(p, t) \geq D(p, t), \forall(p, t)$

$\operatorname{Cap}_{-} \operatorname{Min}_{-} E R P(p, t) \leq E R P(p, t) \geq \operatorname{Cap}_{-} \operatorname{Max}_{-} E R P(p, t), \forall(p, t)$

$N u m \_p c(p c, p) * Z(p, t)=X 1(p c, p, t)+R(p c, p, t)$ 


$$
\begin{aligned}
& \sum_{J} \text { Cavidades }(p c) * \text { Tarefa }(p c, j) * X(p c, j)=\sum_{p} X 1(p c, p, t)+X 0(p c, p, t) \forall t \\
& X(p c, j) \leq \mathrm{Y}(\mathrm{j}) \\
& \text { Cap_Min_EPC }(p c, t) \leq E P C(p c, t) \geq \operatorname{Cap}_{-} \operatorname{Max} E P C(p, t), \forall(p, t) \\
& E R P(p, t-1)+Z(p, t)-S(d, p)=E R P(p, t) \\
& E P C(p c, p, t-1)+X 0(p c, p, t)-R(p c, p, t)=E P C(p c, p, t) \\
& \sum_{P, T}(X 0(p c, p, t)+X(p c, p, t)) * X(p c, j) \leq \operatorname{Cap}_{-} I N J(p c, j) \forall \mathrm{j} \\
& \sum_{t} Z(p, t) \leq C a p_{-} M T(p c) \\
& \sum_{t} Z(p, t) \leq C a p_{-} I M(p) \\
& Z(p, t), S(p, d), X 1(i, j), X 0(i, j), E P R(p, t), E P C(p c, t) \text { inteiro } \geq 0 \\
& X(i, j), Y(j)=\{0,1\}
\end{aligned}
$$

A restrição (1) garante a satisfação da demanda. A restrição (2) e (6) garantem os limites inferiores e superiores para os níveis de estoque, tanto dos produtos finalizados quanto das pecas injetadas. A restrição (3) define a relação linear entre os produtos acabados e as peças que cada um dos produtos para demanda. A restrição (4) define a relação entre o número total de demanda por peças injetadas, os moldes, as cavidades e as tarefas. A restrição (5) garante que uma peça só pode ser injetada se existir uma injetora alocada a um molde adequado a ela. As restrições (7) e (8) garantem a relação e a conservação de fluxo dos estoques dos produtos e das peças, entre dois períodos sequenciais. As restrições (9), (10) e (11) representam as seguintes capacidades: das injetoras, da mão de obra e da impressão, respectivamente. Finalmente, a restrição (12) garante a natureza das variáveis.

\section{IMPLEMENTAÇÃO COMPUTACIONAL E RESULTADOS}

O AIMMS (Advanced Integrated Multidimensional Modeling Software) oferece um ambiente de desenvolvimento, no qual as pessoas experientes em modelagem podem criar aplicações funcionais, prontas para serem usadas por pessoas mais leigas ou usuários finais (ver Ignácio [11]). Como um ambiente de desenvolvimento de apoio analítico à decisão, o AIMMS possibilita uma combinação de características de métodos matemáticos e de ferramentas de desenvolvimento, tais como: o explorador de modelos num ambiente gráfico para se construir e se manter aplicações de modelagem complexa; os métodos de solução exata; os procedimentos que permitem interagir com as linguagens $\mathrm{C}, \mathrm{C}++$, Fortran e ferramentas de interface com as bases de dados através de ODBC/OLE DB.

Este tipo de ferramenta permite implementar, de forma satisfatória, o modelo apresentado, na seção 3. A facilidade com que a ferramenta permite parametrizar os diversos solvers disponíveis, permitiu a resolução do problema o qual, por ser de programação inteira mista teve um tempo computacional de 8900 segundos, utilizando-se $2661 \mathrm{Mb}$ de memória, em um computador com $4 \mathrm{~Gb}$ de memória e processador Intel Core 2 Duo. O solver utilizado denomina-se CPLEX 12.5.

O problema a ser resolvido no presente trabalho, se constitui de 15 produtos eletrodomésticos, tais como: ventiladores, liquidificadores, circuladores de ar, batedeiras etc., dentro de: um horizonte de planejamento de 12 meses; um conjunto de 109 tipos diferentes de moldes; e um parque com 50 injetoras de diversas capacidades e custos de energia e de setup levantados. Os resultados computacionais foram comparados com os resultados reais de planejamento de uma empresa do setor.

Diversos cenários foram rodados e, a seguir é apresentado um cenário base que considera a estrutura produtiva comparada com o planejamento realizado pela equipe sem o uso de modelos matemáticos. A consolidação dos elementos de custos, tais como: o 
faturamento da demanda, custo total de produção, custos de matéria-prima, custos de setup, custos de energia, custos de estoque de peças são resultados do modelo. Dentre os custos mais importantes, destacam-se os custos de estoque, os custos energia e os custos de setup que chegam a ter uma redução de, aproximadamente, $45 \%$ do valor praticado pela empresa.

A seguir são mostradas as características de produtos mais importantes, considerando-se o faturamento. Estes produtos podem ser vistos na Figura 7. Em amarelo é apresentada a demanda por produtos, enquanto em cor azul é apresentado o planejamento realizado pelo modelo matemático e em vermelho, o planejamento sem o uso do modelo matemático.

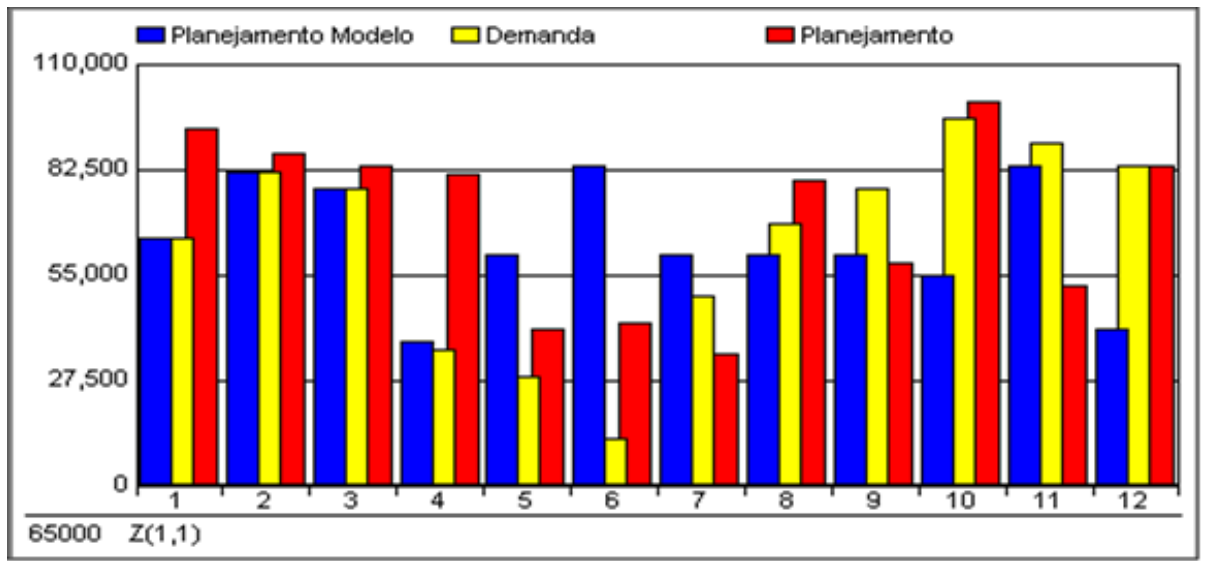

Figura 7 - Produtos acabados - ventiladores

Pode-se notar neste resultado que, nos meses 4, 5, 6 e 7, se apresenta uma produção muito maior que a demandada e a planejada. Nos meses 10 e 11 esta produção se encontra em níveis inferiores que as quantidades produzidas. Isto mostra que a política de produção sem o modelo matemático é bastante diferente da política de produção apresentada, resultante da aplicação do modelo proposto. Isso pode ser visto na Figura 8.

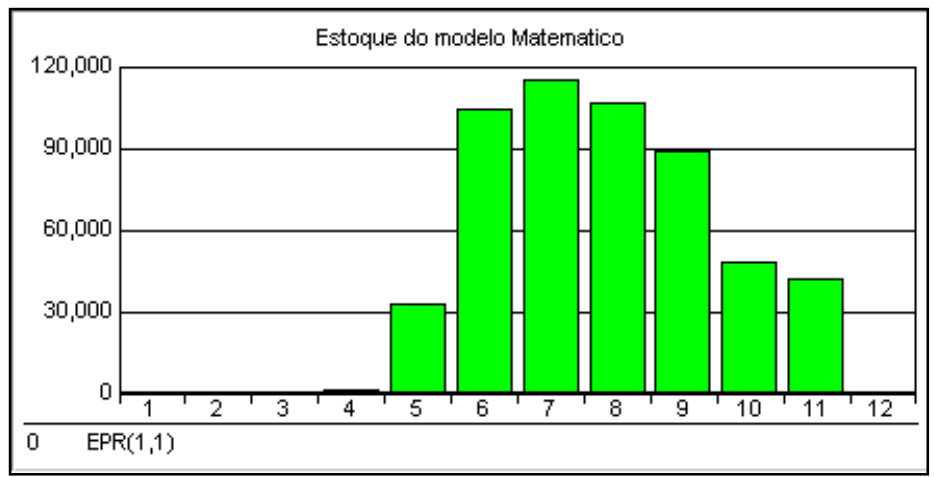

Figura 8. Estoque do planejamento com o modelo matemático

Essa diferença pode ser vista também na Figura 9, que mostra a política de estoque dos produtos sem o uso de modelo matemático.

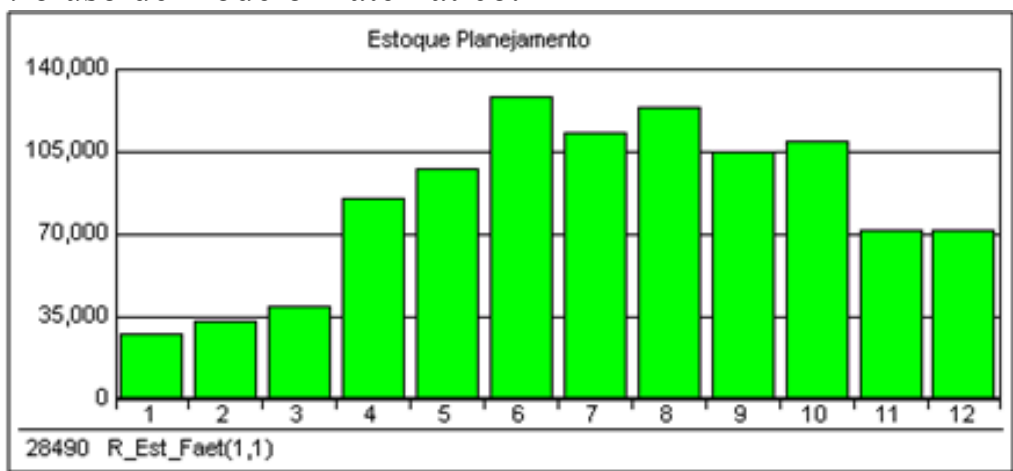

Figura 9 - Estoque do planejamento sem o modelo matemático

A seguir é mostrado o uso de cada uma das injetoras durante os 12 meses de 
planejamento da demanda. Estas informações permitem categorizar o grupo de injetoras pelo uso dentro do horizonte de planejamento.

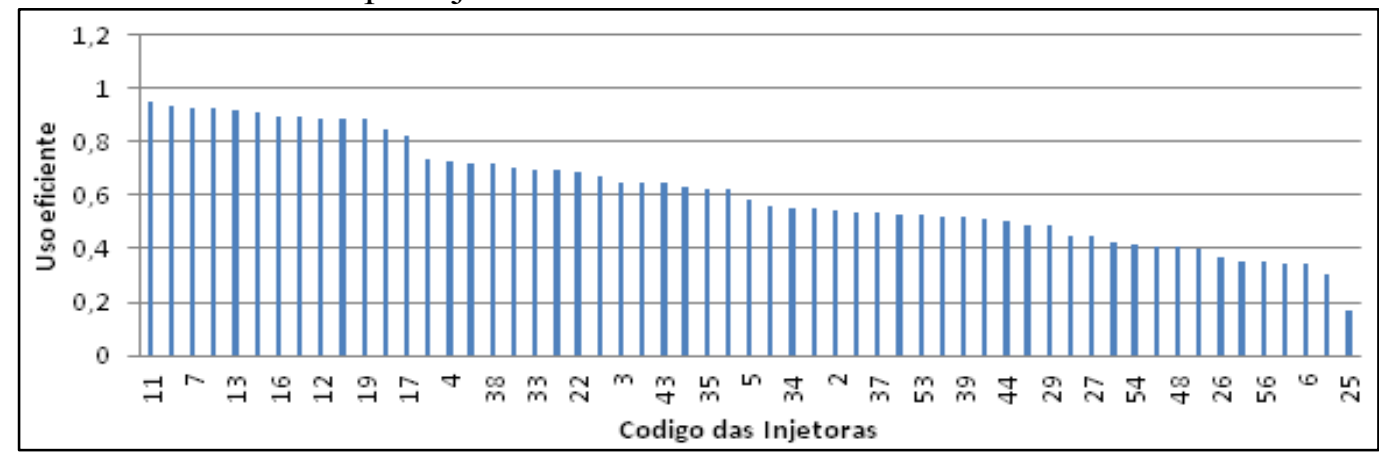

Figura 10 - Uso eficiente das injetoras, no horizonte de planejamento de 12 meses

$\mathrm{Na}$ Figura 10 é mostrado o uso de cada uma das injetoras em termos de tempo disponível por mês, das quais 13 injetoras apresentaram um uso médio acima de $82 \%$, enquanto que 28 injetoras apresentaram um uso médio entre $50 \%$ e $82 \%$ e 16 injetoras apresentaram um uso médio abaixo de 50\%. Deve-se notar que existem 6 injetoras que apresentam um uso inferior a 35\%, mostrando que, em média, das 488 horas disponíveis por mês, somente 156 horas são utilizadas, ficando aproximadamente 291 horas inoperantes.

$\mathrm{O}$ resultado do modelo também fornece o uso eficiente, em cada um dos 12 meses planejados, como por ser visto na Tabela 1.

\begin{tabular}{|c|c|c|c|c|c|c|c|c|c|c|c|c|}
\hline & \multicolumn{12}{|c|}{ Meses } \\
\hline $\begin{array}{l}\text { Cod } \\
\text { Injetora }\end{array}$ & 1 & 2 & 3 & 4 & 5 & 6 & 7 & 8 & 9 & 10 & 11 & 12 \\
\hline 11 & $88 \%$ & $94 \%$ & $98 \%$ & (- $74 \%$ & $89 \%$ & $100 \%$ & $100 \%$ & $100 \%$ & $100 \%$ & $100 \%$ & $100 \%$ & $100 \%$ \\
\hline 14 & $100 \%$ & $90 \%$ & $100 \%$ & $92 \%$ & (-) $39 \%$ & $100 \%$ & $100 \%$ & $100 \%$ & $100 \%$ & $100 \%$ & $100 \%$ & $100 \%$ \\
\hline 7 & $100 \%$ & $84 \%$ & $94 \%$ & (e $64 \%$ & $73 \%$ & $100 \%$ & $100 \%$ & $100 \%$ & $100 \%$ & $100 \%$ & $100 \%$ & $100 \%$ \\
\hline 8 & $97 \%$ & $100 \%$ & $74 \%$ & $97 \%$ & (1) $45 \%$ & $96 \%$ & $100 \%$ & $100 \%$ & $100 \%$ & $100 \%$ & $100 \%$ & $100 \%$ \\
\hline 13 & $99 \%$ & (1) $67 \%$ & $100 \%$ & $80 \%$ & (1) $52 \%$ & $100 \%$ & $100 \%$ & $100 \%$ & $100 \%$ & $100 \%$ & $100 \%$ & $100 \%$ \\
\hline 18 & $100 \%$ & $100 \%$ & $94 \%$ & $0 \%$ & $97 \%$ & $100 \%$ & $100 \%$ & $100 \%$ & $100 \%$ & $100 \%$ & $100 \%$ & $100 \%$ \\
\hline 16 & $100 \%$ & $83 \%$ & $84 \%$ & $100 \%$ & $84 \%$ & $27 \%$ & $100 \%$ & $100 \%$ & $100 \%$ & $100 \%$ & $100 \%$ & $100 \%$ \\
\hline 10 & $100 \%$ & (1) $47 \%$ & $100 \%$ & $87 \%$ & (1) $48 \%$ & $92 \%$ & $100 \%$ & $100 \%$ & $100 \%$ & $100 \%$ & $100 \%$ & $100 \%$ \\
\hline 12 & $91 \%$ & $100 \%$ & $90 \%$ & $58 \%$ & (7) $39 \%$ & $90 \%$ & $100 \%$ & $100 \%$ & $100 \%$ & $100 \%$ & $100 \%$ & $100 \%$ \\
\hline 9 & $89 \%$ & $100 \%$ & $87 \%$ & $91 \%$ & O $17 \%$ & $82 \%$ & $100 \%$ & $100 \%$ & $100 \%$ & $100 \%$ & $100 \%$ & $100 \%$ \\
\hline 19 & (1) $50 \%$ & (1) $56 \%$ & $100 \%$ & $92 \%$ & $65 \%$ & $98 \%$ & $100 \%$ & $100 \%$ & $100 \%$ & $100 \%$ & $100 \%$ & $100 \%$ \\
\hline 15 & $94 \%$ & ( $78 \%$ & () $71 \%$ & (1) $50 \%$ & $30 \%$ & $98 \%$ & $100 \%$ & $100 \%$ & $100 \%$ & $100 \%$ & $100 \%$ & $100 \%$ \\
\hline 17 & $70 \%$ & $100 \%$ & $100 \%$ & (1) $50 \%$ & (1) $44 \%$ & (- $29 \%$ & $100 \%$ & $100 \%$ & $100 \%$ & $100 \%$ & $100 \%$ & $100 \%$ \\
\hline 1 & - $77 \%$ & $94 \%$ & $99 \%$ & $0 \%$ & (16\% & (- $31 \%$ & (5) $79 \%$ & $61 \%$ & $83 \%$ & $100 \%$ & $100 \%$ & $100 \%$ \\
\hline 4 & $100 \%$ & $100 \%$ & $47 \%$ & $80 \%$ & (1) $47 \%$ & $9 \%$ & O $17 \%$ & (. $76 \%$ & $100 \%$ & $100 \%$ & $100 \%$ & $100 \%$ \\
\hline 31 & $89 \%$ & (1) $44 \%$ & $78 \%$ & $90 \%$ & (1) $52 \%$ & (- $39 \%$ & (. $70 \%$ & $100 \%$ & (- $34 \%$ & $100 \%$ & $76 \%$ & $91 \%$ \\
\hline 31 & $89 \%$ & (1) $44 \%$ & $78 \%$ & $90 \%$ & $52 \%$ & (- $39 \%$ & $70 \%$ & $100 \%$ & (- $34 \%$ & $100 \%$ & (อ $76 \%$ & $91 \%$ \\
\hline
\end{tabular}

Tabela 1 - Injetoras com alto índice de uso

Os matizes de cores que vão do verde, passando pelo amarelo, até chegar ao vermelho, representam o tempo de trabalho das injetoras, em cada mês, a partir das menos operantes, ineficientes ou ociosas, até as mais usadas, eficientes ou operantes. 
As injetoras menos eficientes podem também ser observadas na Tabela 2.

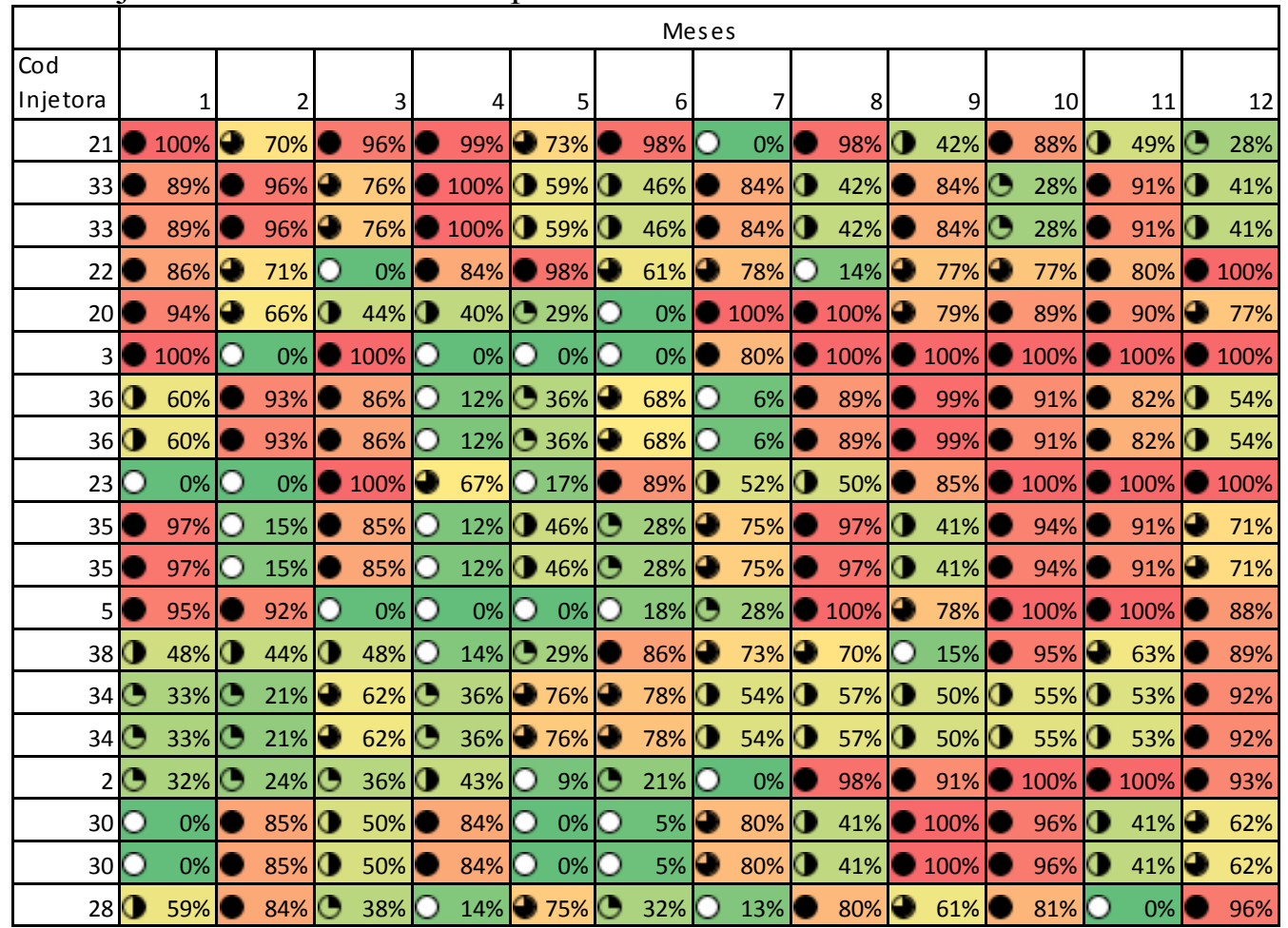

Tabela 2 - Uso de injetoras

A seguir são apresentadas na Tabela 3, as injetoras menos eficientes, com o uso médio anual menor do que $52 \%$.

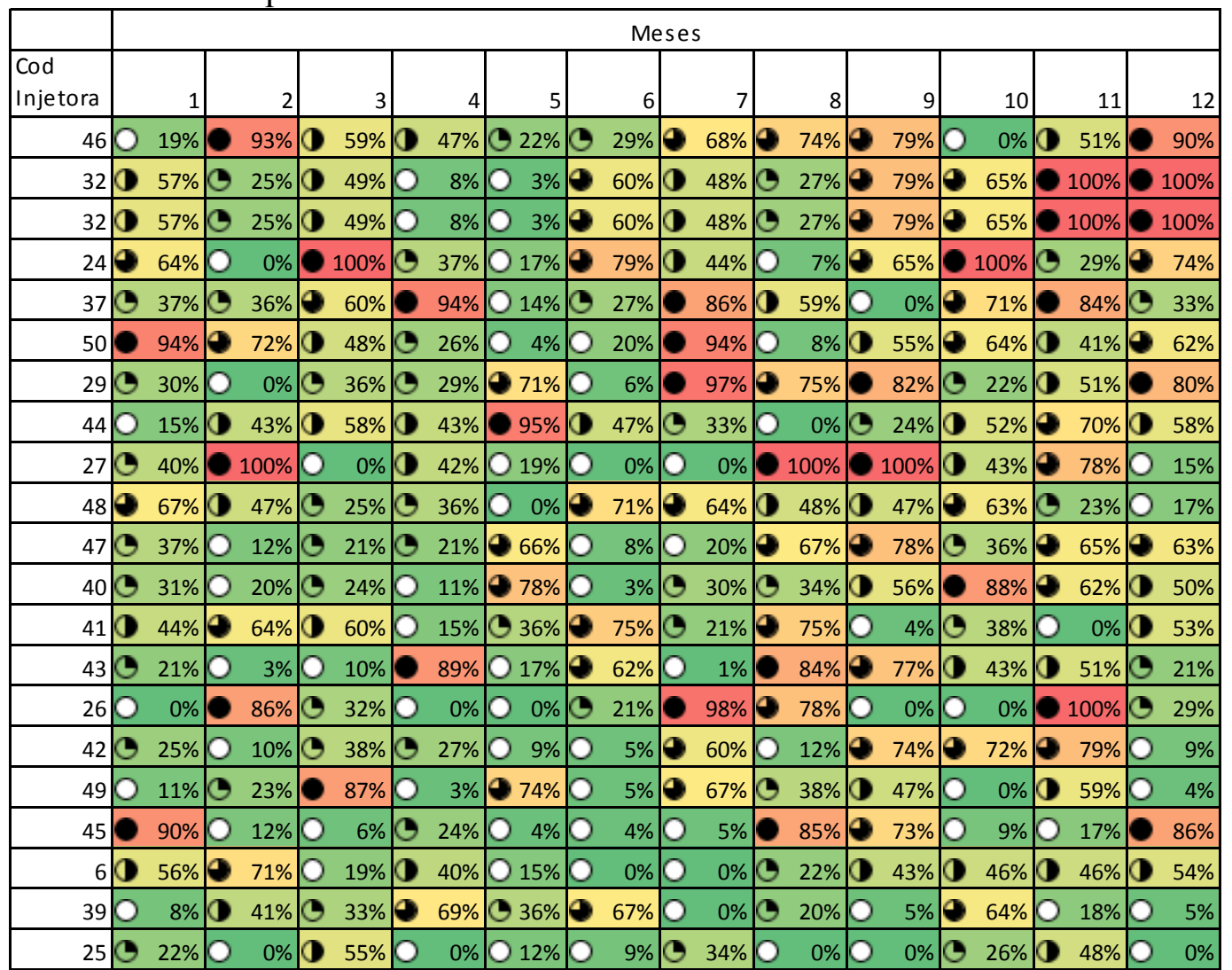

Tabela 3 - Injetoras menos eficientes

\section{CONCLUSÕES DO TRABALHO}

O modelo apresentado no presente artigo propõe uma programação alternativa de produção para a indústria de eletrodomésticos. Pela complexidade dos sistemas produtivos 
neste segmento a ferramenta utilizada permite implementar em diversos cenários, o planejamento da produção e ver o impacto do mesmo, na estrutura real da produção.

A tentativa de acompanhar as demandas por cada um dos produtos não é o modo mais inteligente e eficiente de se minimizar os custos. É muito difícil de visualizar o melhor resultado das previsões planejadas, uma vez que os custos de estoque e os custos de setup definem os equilíbrios de uma configuração ótima que deve ser considerada.

Os resultados do modelo aplicado permitem identificar ineficiências dentro da estrutura produtiva de eletrodomésticos, no que diz respeito ao uso das injetoras e dos moldes. Substituições destes equipamentos podem e devem ser feitas, desde que estas sejam cuidadosamente planejadas e se tenha a real previsão do impacto da satisfação da demanda. Identificar injetoras ineficientes leva ao questionamento de quais delas podem primeiro ser substituídas. Tais questões muitas vezes são difíceis de serem equacionadas, devido à complexidade de se combinar muitos produtos, peças, moldes e injetoras, dentro de um horizonte de planejamento de produção.

A ferramenta apresentada no presente artigo permite a montagem de diversos cenários no planejamento de produção, o que pode ajudar na avaliação e definição dos cenários referentes à substituição de injetoras e de moldes.

Cabe ressaltar que o problema apresenta uma complexidade computacional alta, para qual se deve ter alternativas de solução, em termos de softwares disponíveis, tais como por exemplo o GUROBI ou o desenvolvimento de uma heurística de solução.

\section{REFERÊNCIAS BIBLIOGRÁFICAS}

[1] Souza, A.; Clemente, A. Decisões financeiras e análise de investimentos: fundamentos, técnicas e aplicações. 4. ed. São Paulo: Atlas, 2001.

[2] Hirschfeld, H. Engenharia Econômica e Análise de Custos. 7. ed. São Paulo: Editora Atlas S.A., 2000.

[3] Fleischmann, B.. The discrete lot-sizing and scheduling problem with sequencedependent setup costs. European Journal of Operational Research, 75, 395-404, 1994.

[4] Manrich, S. Processamento de Termoplásticos, Artliber Editora Ltda, 2005.

[5] Haase, K. \& Kimms, A.. Lot sizing and scheduling with sequence dependent setup costs and times and efficient rescheduling opportunities. International Journal of Production Economics, 66, 159-169, 2000.

[6] Meyr, H. Simultaneous lotsizing and scheduling by combining local search with dual reoptimization. European Journal of Operational Research, 120, 311-326, 2000.

[7] Meyr, H. Simultaneous lotsizing and scheduling on parallel machines. European Journal of Operational Research, 139, 277-292, 2002.

[8] Carreno, J.J. Economic lot scheduling for multiple products on parallel identical processors. Management Science, 36, 348-358, 1990.

[9] De Matta, R. \& Guignard, M. The performance of rolling production schedules in a process industry. IIE Transactions, 27, 564-573 (1995).

[10] Clark, A.R. \& Clark, S.J. Rolling-horizon lot-sizing when set-up times are sequencedependent. International Journal of Production Research (2000), 38(10), 2287-2307.

[11] Ignácio, A.A.V.; Ferreira Filho, V.J.M. O Uso de Software de Modelagem AIMMS na Solução de Problemas de Programação Matemática. Pesquisa Operacional, Rio de janeiro, Rio de Janeiro, v. 24, n. 1, p. 197-210, 2004. 